\title{
New Effective Bounds for the Approximate Common Fixed Points and Asymptotic Regularity of Nonexpansive Semigroups
}

\author{
ANGELIKI KOUTSOUKOU-ARGYRAKI
}

\begin{abstract}
We give an explicit, computable and uniform bound for the computation of approximate common fixed points of one-parameter nonexpansive semigroups on a subset $C$ of a Banach space, by proof mining on a proof by Suzuki. The bound obtained here is different to the bound obtained in a very recent work by Kohlenbach and the author which had been derived by proof mining on the -completely differentproof of a generalized version of the particular theorem by Suzuki. We give an adaptation of a logical metatheorem by Gerhardy and Kohlenbach for the given mathematical context, illustrating how the extractability of a computable bound is guaranteed. For uniformly convex $C$, as a corollary to our result we moreover give a computable rate of asymptotic regularity with respect to Kuhfittig's classical iteration schema, by applying a theorem by Khan and Kohlenbach.
\end{abstract}

2010 Mathematics Subject Classification 47H20 (primary); 03F03 (secondary)

Keywords: proof mining, fixed point theory, nonexpansive semigroup, rate of asymptotic regularity, Kuhfittig iteration, computable bound extraction

\section{Introduction}

Proof mining is a research program in applied proof theory originally initiated by Georg Kreisel in the 1950's under the name unwinding of proofs (see [16] or [17]), after he suggested a shift of focus for the application of proof interpretations: from producing relative consistency proofs for foundational purposes to a tool for extracting constructive information from actual mathematical proofs. The program involves the extraction of new quantitative constructive information by logical analysis even of proofs that appear to be nonconstructive. This information is "hidden" behind an implicit use of quantifiers in the proof, and its extraction is guaranteed by certain logical metatheorems (based on variations of Gödel's functional Dialectica interpretation [3]), see for example Kohlenbach [9], provided that the statement proved is of a certain logical form and the assumptions and general mathematical setup fit a specific formal framework. Starting in 
[6], proof mining has been applied by Kohlenbach and his collaborators to various fields of Mathematics, including approximation theory, ergodic theory, fixed point theory, nonlinear analysis and recently PDE theory (Kohlenbach and Koutsoukou-Argyraki [12]. For a review of proof mining results until 2008 we refer to the book Kohlenbach [10] and after 2008 to Kohlenbach [11].

This work is a new contribution of proof mining to nonlinear analysis and fixed point theory ${ }^{1}$. In this paper we obtain effective, quantitative results on the approximate common fixed points of a one-parameter nonexpansive semigroup $\{T(t): t \geq 0\}$ on a subset $C$ of a Banach space $X$ by logical analysis of the proof of a result by Suzuki in [20]. It is very interesting that the bound obtained here (Section 3) is completely different to the bound obtained in another recent work [13] by Kohlenbach and the author. The latter had been derived by proof mining on a proof of a statement again by Suzuki in [21] concerning, again, the common fixed points of $\{T(t): t \geq 0\}$ that is a generalization of the corresponding statement in [20]. We briefly discuss a comparison between the bound in [13] and the one obtained in this work. In Section 3 we will also present an adaptation of a general logical metatheorem by Gerhardy and Kohlenbach for the specific mathematical framework and the assumptions by Suzuki, in order to illustrate how the metatheorem guarantees the extractability of the bound in the situation at hand. In particular we write the metatheorem adapted for an intermediate result. We can write such an adaptation as all the input data is majorizable and all assumptions can be written as universal statements. The metatheorem predicts that a computable bound can be extracted which will be highly uniform, ie it will depend on general bounding information on the input data (majorants), and on nothing else. Indeed we do extract such a bound via proof mining. In the last section we give a corollary to our result for the case of uniformly convex $C$ : we apply our result to extract a rate of asymptotic regularity for $\{T(t): t \geq 0\}$ with respect to a classical iteration schema introduced by Kuhfittig in [18]. This is achieved by making use of a theorem by Khan and Kohlenbach [5] which was derived via proof mining on the proof of a result by Kuhfittig [18].

\section{Preliminaries}

By $\mathbb{N}$ we denote the set of natural numbers $\{1,2, \ldots\}$, by $\mathbb{Z}$ the set of integers and by $\mathbb{Z}^{+}, \mathbb{Q}^{+}, \mathbb{R}^{+}$the sets of nonnegative integers, rationals and reals respectively. For $x \in \mathbb{R}$ (following the notation in [20]) by $[x] \in \mathbb{Z}$ we denote the floor function, ie the

\footnotetext{
${ }^{1}$ The results here are also included in the author's $\mathrm{PhD}$ thesis [14].
} 
largest integer not exceeding $x$. Moreover, by $\lceil x\rceil \in \mathbb{Z}$ we denote the ceiling function, ie the smallest integer exceeding $x$ or equal to $x$.

Definition 2.1 Given a Banach space $X$ and $C \subseteq X$, a mapping $T$ on $C$ is nonexpansive if

$$
\forall x, y \in C\|T x-T y\| \leq\|x-y\| .
$$

Definition 2.2 A family $\{T(t): t \geq 0\}$ of self-mappings $T(t): C \rightarrow C$ for a subset $C$ of a Banach space $X$ is called a one-parameter strongly continuous semigroup of nonexpansive mappings (or nonexpansive semigroup for short) if the following conditions hold:

(1) for all $t \geq 0, T(t)$ is a nonexpansive mapping on $C$,

(2) $T(s) \circ T(t)=T(s+t)$,

(3) for each $x \in C$, the mapping $t \rightarrow T(t) x$ from $[0, \infty)$ into $C$ is continuous.

In the following we will need to make use of the concepts of uniform equicontinuity for a nonexpansive semigroup and modulus of uniform equicontinuity introduced in [13]:

Definition 2.3 (Kohlenbach and Koutsoukou-Argyraki [13]) We say that a nonexpansive semigroup $\{T(t): t \geq 0\}$ on a subset $C$ of a Banach space $X$ is uniformly equicontinuous if the mapping $t \rightarrow T(t) z$ is uniformly continuous on each compact interval $[0, K]$ for all $K \in \mathbb{N}$ and given a $b \in \mathbb{N}$ it has a common modulus of continuity for all $z \in C_{b}$. Namely if there exists a function $\omega: \mathbb{N} \times \mathbb{N} \times \mathbb{N} \rightarrow \mathbb{N}$ so that

$$
\begin{aligned}
\forall b \in \mathbb{N} \forall z \in C_{b} \forall m \in \mathbb{N} \forall K \in \mathbb{N} \forall t, t^{\prime} \in & {[0, K] } \\
& \left(\left|t-t^{\prime}\right|<2^{-\omega_{K, b}(m)} \rightarrow\left\|T(t) z-T\left(t^{\prime}\right) z\right\|<2^{-m}\right)
\end{aligned}
$$

where $C_{b}:=\{z \in C:\|z\| \leq b\}$. We call $\omega$ a modulus of uniform equicontinuity for the nonexpansive semigroup $\{T(t): t \geq 0\}$.

For our bound extractions we will assume uniform equicontinuity as defined above for the nonexpansive semigroup $\{T(t): t \geq 0\}$. The motivation from introducing equicontinuity, ie the property of having a common modulus of continuity for all $z$ that are norm-bounded by a specific $b \in \mathbb{N}$, and assuming this requirement for our semigroup, comes from the need to fit the framework of the logical metatheorems that will guarantee the extractability of the bounds as in order to achieve the majorizability of the semigroup equicontinuity is required. This slight strengthening is harmless as in praxis one may a posteriori remove equicontinuity but then the bound would be less 
uniform as it would depend on each point which would not be desirable. Moreover it would then not be possible to obtain the results on asymptotic regularity.

In the literature one may find several examples where uniform equicontinuity is fulfilled, for instance see [13]. Moreover, any nonexpansive semigroup generated from a bounded accretive operator via the Crandall-Liggett formula fulfills the property of uniform equicontinuity, as can been seen in Crandall and Liggett [2] (see in particular (1.11) there).

Because, as we will later see, in Suzuki [20] an irrationality assumption is made, we will need a quantitative version of this assumption in our quantitative analysis of his proof. For that we will make use of the following:

Let $\gamma \in \mathbb{R}^{+} \backslash \mathbb{Q}^{+}$. Then

$$
\forall p \in \mathbb{N} \forall p^{\prime} \in \mathbb{Z}^{+} \exists z \in \mathbb{N}\left(\left|\gamma-\frac{p^{\prime}}{p}\right| \geq \frac{1}{z}\right) .
$$

The Skolem normal form of the above is

$$
\exists f_{\gamma}: \mathbb{N} \times \mathbb{Z}^{+} \rightarrow \mathbb{N} \forall p \in \mathbb{N} \forall p^{\prime} \in \mathbb{Z}^{+}\left(\left|\gamma-\frac{p^{\prime}}{p}\right| \geq \frac{1}{f_{\gamma}\left(p, p^{\prime}\right)}\right)
$$

and $f_{\gamma}$ is the corresponding Skolem function.

Definition 2.4 The function $f_{\gamma}$ as in (i) above is called an effective irrationality measure for $\gamma$.

\section{Proof Mining in Praxis, a Metatheorem Adaptation and Results}

Our main result is a quantitative version of the following result by Suzuki:

Theorem 3.1 (Suzuki [20, Proposition 2]) Let $X$ be a Banach space and let $\{T(t): t \geq$ $0\}$ be a one-parameter nonexpansive semigroup on a subset $C$ of $X$. Let $\alpha, \beta$ be positive real numbers so that $\alpha / \beta \in \mathbb{R} \backslash \mathbb{Q}$. Then we have

$$
\bigcap_{t \geq 0} F(T(t))=F(T(\alpha)) \cap F(T(\beta)) .
$$

The inclusion

$$
\bigcap_{t \geq 0} F(T(t)) \subseteq F(T(\alpha)) \cap F(T(\beta))
$$


is trivial, so in the proof of Theorem 3.1 in [20] the nontrivial inclusion

$$
\bigcap_{t \geq 0} F(T(t)) \supseteq F(T(\alpha)) \cap F(T(\beta))
$$

is proved. Our main result which we will show here, constitutes in particular a quantitative version of the latter statement. As this can be written as

$$
\begin{aligned}
\forall z \in C(\forall m \in \mathbb{N}\|T(\alpha) z-z\| \leq & 2^{-m} \wedge\|T(\beta) z-z\| \leq 2^{-m} \rightarrow \\
& \left.\forall k \in \mathbb{N} \forall M \in \mathbb{N} \forall t \in[0, M]\|T(t) z-z\|<2^{-k}\right)
\end{aligned}
$$

ie (by prenexation)

$$
\begin{aligned}
\forall z \in C \forall k \in & \mathbb{N} \forall M \in \mathbb{N} \forall t \in[0, M] \exists m \in \mathbb{N} \\
& \left(\|T(\alpha) z-z\| \leq 2^{-m} \wedge\|T(\beta) z-z\| \leq 2^{-m} \rightarrow\|T(t) z-z\|<2^{-k}\right),
\end{aligned}
$$

we have a $\forall \exists(\forall \rightarrow \exists)$ ie $\forall \exists$ statement, so it is possible to extract a computable bound on $m \in \mathbb{N}$ (see Kohlenbach [10]). This will be done by performing proof mining on Suzuki's proof of the above.

Before the main result we will firstly obtain quantitative versions of Proposition 1 and Lemma 3 in [20] by proof mining on Suzuki's proofs.

Proposition 3.2 (Suzuki [20, Proposition 1]) Let $X$ be a Banach space and let $\{T(t): t \geq 0\}$ be a one-parameter nonexpansive semigroup on $C \subseteq X$. Let $\left\{\alpha_{n}\right\}$ be a sequence in $[0, \infty)$ converging to $\alpha_{\infty} \in[0, \infty)$, and satisfying $\alpha_{n} \neq \alpha_{\infty}$ for all $n \in \mathbb{N}$. Suppose that $z \in C$ satisfies $T\left(\alpha_{n}\right) z=z$ for all $n \in \mathbb{N}$. Then $z$ is a common fixed point of $\{T(t): t \geq 0\}$.

A formalized version of the above statement is:

$$
\forall z \in C\left(\forall \delta>0 \forall n \in \mathbb{N}\left\|T\left(\alpha_{n}\right) z-z\right\| \leq \delta \rightarrow \forall k \in \mathbb{N} \forall t \in[0, \infty)\|T(t) z-z\|<2^{-k}\right)
$$

By prenexing the above we have

$$
\begin{aligned}
\forall z \in C \forall k \in \mathbb{N} \forall t \in[0, \infty) \exists \delta>0 & \exists n \in \mathbb{N} \\
& \left(\left\|T\left(\alpha_{n}\right) z-z\right\| \leq \delta \rightarrow\|T(t) z-z\|<2^{-k}\right),
\end{aligned}
$$

ie (setting $\left.C_{b}:=\{z \in C:\|z\| \leq b\}\right)$

( $\triangle) \quad \forall b \in \mathbb{N} \forall z \in C_{b} \forall k \in \mathbb{N} \forall M \in \mathbb{N} \forall t \in[0, M] \exists \delta>0 \exists n \in \mathbb{N}$

$$
\left(\left\|T\left(\alpha_{n}\right) z-z\right\| \leq \delta \rightarrow\|T(t) z-z\|<2^{-k}\right) .
$$


The goal is to extract computable bounds on $\delta$ and $n$ from the proof of the statement. This bound extraction would then amount to obtaining a quantitative version of the statement.

We can show that we may write a version of a general logical metatheorem by Gerhardy and Kohlenbach (see [10, Theorem 17.52 and Corollary 17.71]) adapted in particular explicitly for the mathematical setting and assumptions of Suzuki's proposition above. Note that by $\mathcal{A}^{\omega}[X,\|\cdot\|, C]_{-b}$ we denote the theory involving the system $\mathcal{A}^{\omega}$, defined as $\mathcal{A}^{\omega}: \equiv \mathrm{WE}^{\mathrm{PA}}{ }^{\omega}+\mathrm{QF}-\mathrm{AC}+\mathrm{WKL}$ (denoting the weakly extensional Peano arithmetic in all finite types, the quantifier-free axiom of choice and weak König's lemma respectively), where the type system is extended over two ground types $\mathbb{N}, X$ for a normed space $X$ (see [10]). Our adaptation reads:

Metatheorem 3.3 Assume that we have a proof of a sentence in $\mathcal{A}^{\omega}[X,\|\cdot\|, C]_{-b}$ :

$$
\begin{aligned}
& \forall t \in \mathbb{R}^{+} \forall z \in C \forall T \in C \times \mathbb{R}^{+} \rightarrow C \forall\left\{\alpha_{n}\right\} \subseteq \mathbb{R}^{+} \forall \alpha_{\infty} \in \mathbb{R}^{+} \\
& \forall \omega \in \mathbb{N} \times \mathbb{N} \times \mathbb{N} \rightarrow \mathbb{N} \forall \Phi, \Psi \in \mathbb{N} \rightarrow \mathbb{N} \forall m \in \mathbb{N} \exists k \in \mathbb{N} \exists n \in \mathbb{N} \\
&\left(\left(\forall t \in \mathbb{R}^{+} \forall x, y \in C\|T(t) x-T(t) y\| \leq_{\mathbb{R}}\|x-y\|\right)\right. \\
& \wedge\left(\forall x \in C \forall t, s \in \mathbb{R}^{+} T(s) \circ T(t)(x)=_{X} T(s+t)(x)\right) \\
& \wedge\left(\forall b \in \mathbb{N} \forall q \in C \forall m \in \mathbb{N} \forall K \in \mathbb{N} \forall t, t^{\prime} \in[0, K]\right. \\
&\left.\quad\left(\|q\|<_{\mathbb{R}} b \wedge\left|t-t^{\prime}\right|<_{\mathbb{R}} 2^{-\omega_{K, b}(m)} \rightarrow\left\|T(t) q-T\left(t^{\prime}\right) q\right\| \leq_{\mathbb{R}} 2^{-m}\right)\right) \\
& \wedge\left(\forall n \in \mathbb{N}\left|\alpha_{n}-\alpha_{\infty}\right| \geq_{\mathbb{R}} 2^{-\Psi(n)}\right) \\
& \wedge\left(\forall k \in \mathbb{N} \forall n \geq \Phi(k)\left|\alpha_{n}-\alpha_{\infty}\right| \leq_{\mathbb{R}} 2^{-k}\right) \\
&\left.\wedge\left(\left\|T\left(\alpha_{n}\right) z-z\right\| \leq_{\mathbb{R}} 2^{-k}\right) \rightarrow\|T(t) z-z\|<_{\mathbb{R}} 2^{-m}\right)
\end{aligned}
$$

Then one can extract from the proof primitive recursive in the sense of Gödel functionals $W, \tilde{W}$ so that

$$
\begin{aligned}
& \forall M \in \mathbb{N} \forall t \in[0, M] \forall L \in \mathbb{N} \forall\left\{\alpha_{n}\right\} \in[0, L]^{\mathbb{N}} \forall \alpha_{\infty} \in[0, L] \forall B \in \mathbb{N} \forall z \in C_{B} \\
& \forall T \in C \times \mathbb{R}^{+} \rightarrow C \forall \omega \in \mathbb{N} \times \mathbb{N} \times \mathbb{N} \rightarrow \mathbb{N} \forall \Phi, \Psi \in \mathbb{N} \rightarrow \mathbb{N} \\
& \forall m \in \mathbb{N} \exists k \leq W\left(B, M, L, \Psi^{\prime}, \Phi^{\prime}, m, \omega^{\prime}\right) \exists n \leq \tilde{W}\left(B, M, L, \Psi^{\prime}, \Phi^{\prime}, m, \omega^{\prime}\right) \\
&\left(\left(\forall t \in \mathbb{R}^{+} \forall x, y \in C\|T(t) x-T(t) y\| \leq_{\mathbb{R}}\|x-y\|\right)\right. \\
& \wedge\left(\forall x \in C \forall t, s \in \mathbb{R}^{+} T(s) \circ T(t)(x)=_{X} T(s+t)(x)\right) \\
& \wedge\left(\forall b \in \mathbb{N} \forall q \in C \forall m \in \mathbb{N} \forall K \in \mathbb{N} \forall t, t^{\prime} \in[0, K]\right. \\
&\left.\quad\left(\|q\|<\mathbb{R} \wedge \wedge\left|t-t^{\prime}\right|<_{\mathbb{R}} 2^{-\omega_{K, b}(m)} \rightarrow\left\|T(t) q-T\left(t^{\prime}\right) q\right\| \leq_{\mathbb{R}} 2^{-m}\right)\right) \\
& \wedge\left(\forall n \in \mathbb{N}\left|\alpha_{n}-\alpha_{\infty}\right| \geq_{\mathbb{R}} 2^{-\Psi(n)}\right)
\end{aligned}
$$




$$
\begin{aligned}
& \wedge\left(\forall k \in \mathbb{N} \forall n \geq \Phi(k)\left|\alpha_{n}-\alpha_{\infty}\right| \leq_{\mathbb{R}} 2^{-k}\right) \\
& \left.\wedge\left(\left\|T\left(\alpha_{n}\right) z-z\right\| \leq_{\mathbb{R}} 2^{-k}\right) \rightarrow\|T(t) z-z\|<_{\mathbb{R}} 2^{-m}\right)
\end{aligned}
$$

holds (in the sense of Kohlenbach [10, Definition 17.68]) for any nontrivial normed space $X$ with a nonempty $C \subseteq X$.

The bound depends only on general bounding information (majorants, see [10, Definition 17.50]) on the input data. It is easy to show that a one-parameter nonexpansive semigroup is majorizable (see Koutsoukou-Argyraki [14]). Here the displacement assumption for some arbitrary element of the sequence is trivially covered because of the premise $\left\|T\left(\alpha_{n}\right) z-z\right\| \leq_{\mathbb{R}} 2^{-k}$. Also note that the input number theoretic functions $\Phi, \Psi, \omega$ are replaced with the nondecreasing functions $\Phi^{\prime}, \Psi^{\prime}, \omega^{\prime}$ so that the latter are their own majorants and we have ensured that all the assumptions introduced are formulated as universal statements. Notice that the bound will not depend on representatives of the sequence of reals $\left\{\alpha_{n}\right\} \subseteq \mathbb{R}^{+}$nor its limit $\alpha_{\infty} \in \mathbb{R}^{+}$but on $L \in \mathbb{N}$ as we can instead write $\forall L \in \mathbb{N} \forall \alpha_{n} \in[0, L]^{\mathbb{N}} \forall \alpha_{\infty} \in[0, L]$ and they can therefore be seen as elements not of the Polish space $\mathbb{R}^{+}$but of the compact spaces $[0, L]^{\mathbb{N}}$ and $[0, L]$ respectively. Therefore the bound will depend only on such a parameter $L \in \mathbb{N}$. This approach has similarly been followed for $t \in \mathbb{R}^{+}$, as instead of $\forall t \in \mathbb{R}^{+}$we write $\forall M \in \mathbb{N} \forall t \in[0, M]$ so that $t$ will be considered an element of the compact space $[0, M]$ and the bound will only depend on the parameter $M \in \mathbb{N}$. The above metatheorem is a direct adaptation of the aforementioned metatheorem by Gerhardy and Kohlenbach, as we have written the assumptions of the above given in Suzuki [20, Proposition 1] as universal statements. In particular, the assertion of Suzuki's proposition is written as a $\forall \exists(\forall \rightarrow \exists)$ ie a $\forall \exists$ statement (considering the representation of real numbers as in [10] according to which statements involving $\leq, \geq,=$ are seen as universal and statements involving $<$,$\rangle are seen as existential). Indeed, as the above metatheorem$ predicts we do succeed to extract a computable and highly uniform bound by performing proof mining on Suzuki's proof. In particular, we obtain the following result (note that $\Psi, \Phi, \omega$ below are as in the above metatheorem- the bound will depend on their majorants $\Psi^{\prime}, \Phi^{\prime}, \omega^{\prime}$ so for notation simplicity one may a priori choose $\Psi, \Phi, \omega$ to be nondecreasing thus identifiable with their majorants $\left.\Psi^{\prime}, \Phi^{\prime}, \omega^{\prime}\right)$ :

Theorem 3.4 (Quantitative version of [20, Proposition 1]) Let $X$ be a Banach space and let $\{T(t): t \geq 0\}$ be a one-parameter uniformly equicontinuous semigroup of nonexpansive mappings on a subset $C$ of $X$, with a modulus of uniform equicontinuity $\omega$. Let $\left\{\alpha_{n}\right\}$ be a sequence of reals in $[0, \infty)$ converging to $\alpha_{\infty} \in[0, \infty)$ with a rate of convergence $\Phi: \mathbb{N} \rightarrow \mathbb{N}$, and so that $\forall n \in \mathbb{N}\left(\left|\alpha_{n}-\alpha_{\infty}\right|>2^{-\Psi(n)}\right)$ where $\Psi: \mathbb{N} \rightarrow \mathbb{N}$. Let $L \in \mathbb{N}$ be such that for all $n \in \mathbb{N}\left\{\alpha_{n}\right\}, \alpha_{\infty} \in[0, L]$. Then 
(*) $\forall k \in \mathbb{N} \forall b \in \mathbb{N} \forall z \in C_{b} \forall M \in \mathbb{N} \forall L \in \mathbb{N} \exists n \leq \tilde{W}$

$$
\left(\left\|T\left(\alpha_{n}\right) z-z\right\| \leq W \rightarrow \forall t \in[0, M]\|T(t) z-z\|<2^{-k}\right)
$$

with

$$
\begin{aligned}
\tilde{W} & =\tilde{W}(k, b, M, L, \Phi, \Psi, \omega) \\
& =\max \left\{\Phi\left(\omega_{b, M+1}(k+1)\right), \Phi\left(\omega_{b, L}\left(k+1+\left\lceil\log _{2}\left(3 M \sum_{i=1}^{\Phi\left(\omega_{b, M+1}(k+1)\right)} 2^{\Psi(i)}\right)\right\rceil\right)\right)\right\}
\end{aligned}
$$

and

$$
W=W(k, b, M, \Phi, \Psi, \omega)=\frac{2^{-(k+1)}}{3 M \sum_{i=1}^{\Phi\left(\omega_{b, M+1}(k+1)\right)} 2^{\Psi(i)}}
$$

Proof For $(\triangle)$, which is of the logical form $\forall \exists$, the above stated metatheorem guarantees the extraction of computable bounds on $\delta$ and $n$. We will extract such bounds by proof mining on Proposition 1 and therefore obtain $(*)$.

As is done in the proof of [20, Proposition 1], we define

$$
\beta_{n}:=\left|\alpha_{n}-\alpha_{\infty}\right|
$$

By this definition, clearly the rate of convergence of $\left\{\beta_{n}\right\}$ to 0 is the same as the rate of convergence $\Phi$ of $\left\{\alpha_{n}\right\}$ to $\alpha_{\infty}$ and, moreover, by the assumption we have

$$
\forall n \in \mathbb{N} \beta_{n}>2^{-\Psi(n)} \text {. }
$$

Now, in both cases $\alpha_{n}=\alpha_{\infty}+\beta_{n}$ and $\alpha_{\infty}=\alpha_{n}+\beta_{n}$ we claim that we have, for all $n \in \mathbb{N}$,

$$
\left\|T\left(\beta_{n}\right) z-z\right\| \leq\left\|T\left(\alpha_{\infty}\right) z-z\right\|+\left\|T\left(\alpha_{n}\right) z-z\right\| .
$$

The above claim follows directly by the semigroup properties and the triangle inequality, ie if $\alpha_{n}=\alpha_{\infty}+\beta_{n}$ we have

$$
\begin{aligned}
\left\|T\left(\beta_{n}\right) z-z\right\| & =\left\|T\left(\beta_{n}\right) z-z+T\left(\alpha_{n}\right) z-T\left(\alpha_{n}\right) z\right\| \\
& \leq\left\|T\left(\beta_{n}\right) z-T\left(\alpha_{n}\right) z\right\|+\left\|T\left(\alpha_{n}\right) z-z\right\| \\
& =\left\|T\left(\beta_{n}\right) z-T\left(\alpha_{\infty}+\beta_{n}\right) z\right\|+\left\|T\left(\alpha_{n}\right) z-z\right\| \\
& =\left\|T\left(\beta_{n}\right) z-T\left(\beta_{n}\right) T\left(\alpha_{\infty}\right) z\right\|+\left\|T\left(\alpha_{n}\right) z-z\right\| \\
& \leq\left\|z-T\left(\alpha_{\infty}\right) z\right\|+\left\|T\left(\alpha_{n}\right) z-z\right\|
\end{aligned}
$$


and analogously if $\alpha_{\infty}=\alpha_{n}+\beta_{n}$ we have

$$
\begin{aligned}
\left\|T\left(\beta_{n}\right) z-z\right\| & =\left\|T\left(\beta_{n}\right) z-z+T\left(\alpha_{\infty}\right) z-T\left(\alpha_{\infty}\right) z\right\| \\
& =\left\|T\left(\beta_{n}\right) z-z+T\left(\alpha_{n}+\beta_{n}\right) z-T\left(\alpha_{n}+\beta_{n}\right) z\right\| \\
& \leq\left\|T\left(\beta_{n}\right) z-T\left(\beta_{n}\right) T\left(\alpha_{n}\right) z\right\|+\left\|T\left(\alpha_{n}+\beta_{n}\right) z-z\right\| \\
& \leq\left\|z-T\left(\alpha_{n}\right) z\right\|+\left\|T\left(\alpha_{\infty}\right) z-z\right\| .
\end{aligned}
$$

Now let $t \in[0, M]$ for some $M \in \mathbb{N}$. By Suzuki [20, Lemma 2], there exists a sequence $\left\{k_{n}\right\} \in \mathbb{N} \cup\{0\}$ (as defined in [20, Lemma 2]) such that $\forall m \in \mathbb{N} \forall n \geq$ $\Phi(m)\left(\left|\sum_{i=1}^{n} k_{i} \beta_{i}-t\right|<2^{-m}\right)$. (Note that as by [20, Lemma 2] we have that for all $n \in \mathbb{N}, 0 \leq t-\sum_{i=1}^{n} k_{i} \beta_{i}<\beta_{n}$, as a rate of convergence of $\left\{\sum_{i=1}^{n} k_{i} \beta_{i}\right\}$ to $t$ we can take the rate of convergence $\Phi$ of $\left\{\beta_{n}\right\}$ to 0 (ie the rate of convergence $\Phi$ of $\left\{\alpha_{n}\right\}$ to $\left.\alpha_{\infty}\right)$.

Now the triangle inequality gives

$$
\begin{aligned}
\|T(t) z-z\| & =\left\|T(t) z-z+T\left(\sum_{i=1}^{n} k_{i} \beta_{i}\right) z-T\left(\sum_{i=1}^{n} k_{i} \beta_{i}\right) z\right\| \\
& \leq\left\|T\left(\sum_{i=1}^{n} k_{i} \beta_{i}\right) z-z\right\|+\left\|T(t) z-T\left(\sum_{i=1}^{n} k_{i} \beta_{i}\right) z\right\| .
\end{aligned}
$$

We moreover have

$$
\begin{aligned}
\| T\left(\sum_{i=1}^{n}\right. & \left.k_{i} \beta_{i}\right) z-z\|=\| T\left(k_{1} \beta_{1}\right) T\left(k_{2} \beta_{2}\right) \ldots T\left(k_{n} \beta_{n}\right) z-z \| \\
& =\left\|T\left(k_{1} \beta_{1}\right) T\left(k_{2} \beta_{2}\right) \ldots T\left(k_{n} \beta_{n}\right) z-z+T\left(k_{1} \beta_{1}\right) z-T\left(k_{1} \beta_{1}\right) z\right\| \\
\leq & \left\|T\left(k_{1} \beta_{1}\right) T\left(k_{2} \beta_{2}\right) \ldots T\left(k_{n} \beta_{n}\right) z-T\left(k_{1} \beta_{1}\right) z\right\|+\left\|T\left(k_{1} \beta_{1}\right) z-z\right\| \\
\leq & \left\|T\left(k_{2} \beta_{2}\right) \ldots T\left(k_{n} \beta_{n}\right) z-z\right\|+\left\|T\left(k_{1} \beta_{1}\right) z-z\right\| \\
= & \left\|T\left(k_{2} \beta_{2}\right) \ldots T\left(k_{n} \beta_{n}\right) z-z\right\|+\left\|T^{k_{1}}\left(\beta_{1}\right) z-z\right\| \\
= & \left\|T\left(k_{2} \beta_{2}\right) \ldots T\left(k_{n} \beta_{n}\right) z-z\right\|+\left\|T^{k_{1}}\left(\beta_{1}\right) z-z+T\left(\beta_{1}\right) z-T\left(\beta_{1}\right) z\right\| \\
\leq & \left\|T\left(k_{2} \beta_{2}\right) \ldots T\left(k_{n} \beta_{n}\right) z-z\right\|+\left\|T^{k_{1}}\left(\beta_{1}\right) z-T\left(\beta_{1}\right) z\right\|+\left\|-z+T\left(\beta_{1}\right) z\right\| \\
\leq & \left\|T\left(k_{2} \beta_{2}\right) \ldots T\left(k_{n} \beta_{n}\right) z-z\right\|+\left\|T^{k_{1}-1}\left(\beta_{1}\right) z-z\right\|+\left\|-z+T\left(\beta_{1}\right) z\right\| \\
\leq & \left\|T\left(k_{2} \beta_{2}\right) \ldots T\left(k_{n} \beta_{n}\right) z-z\right\|+k_{1}\left\|T\left(\beta_{1}\right) z-z\right\| \\
\leq & k_{1}\left\|T\left(\beta_{1}\right) z-z\right\|+k_{2}\left\|T\left(\beta_{2}\right) z-z\right\|+\ldots . k_{n}\left\|T\left(\beta_{n}\right) z-z\right\| \\
\leq & k_{1}\left(\left\|T\left(\alpha_{1}\right) z-z\right\|+\left\|T\left(\alpha_{\infty}\right) z-z\right\|\right) \\
& \quad+\ldots+k_{n}\left(\left\|T\left(\alpha_{n}\right) z-z\right\|+\left\|T\left(\alpha_{\infty}\right) z-z\right\|\right) \\
= & \left(k_{1}\left\|T\left(\alpha_{\infty}\right) z-z\right\|+\ldots+k_{n}\left\|T\left(\alpha_{\infty}\right) z-z\right\|\right)
\end{aligned}
$$




$$
\begin{array}{r}
\quad+\left(k_{1}\left\|T\left(\alpha_{1}\right) z-z\right\|+\ldots+k_{n}\left\|T\left(\alpha_{n}\right) z-z\right\|\right) \\
=\left\|T\left(\alpha_{\infty}\right) z-z\right\| \sum_{i=1}^{n} k_{i}+\sum_{i=1}^{n} k_{i}\left\|T\left(\alpha_{i}\right) z-z\right\|
\end{array}
$$

and by the triangle inequality

$$
\left\|T\left(\alpha_{\infty}\right) z-z\right\| \leq\left\|T\left(\alpha_{m}\right) z-T\left(\alpha_{\infty}\right) z\right\|+\left\|z-T\left(\alpha_{m}\right) z\right\|
$$

for any arbitrary $m \in \mathbb{N}$, so overall we have calculated that

$$
\begin{aligned}
\|T(t) z-z\| \leq\left(\left\|T\left(\alpha_{m}\right) z-T\left(\alpha_{\infty}\right) z\right\|\right. & \left.+\left\|z-T\left(\alpha_{m}\right) z\right\|\right) \sum_{i=1}^{n} k_{i} \\
& +\sum_{i=1}^{n} k_{i}\left\|T\left(\alpha_{i}\right) z-z\right\|+\left\|T(t) z-T\left(\sum_{i=1}^{n} k_{i} \beta_{i}\right) z\right\| .
\end{aligned}
$$

By the construction of [20, Lemma 2], it is

$$
k_{n}=\left[\frac{\delta_{n}}{\beta_{n}}\right], \quad n \in \mathbb{N}
$$

where $\left\{\delta_{n}\right\}$ is a sequence in $[0, \infty)$ defined by $\delta_{1}=t ; \delta_{n+1}=\delta_{n}-k_{n} \beta_{n}$. So, as for all $n \in \mathbb{N}, \delta_{n}-\delta_{n+1}=k_{n} \beta_{n} \geq 0,\left\{\delta_{n}\right\}$ is nonincreasing. Therefore, for all $n \in \mathbb{N}$,

$$
k_{n}=\left[\frac{\delta_{n}}{\beta_{n}}\right] \leq \frac{\delta_{n}}{\beta_{n}} \leq \frac{\delta_{1}}{\beta_{n}}=\frac{t}{\beta_{n}}<t 2^{\Psi(n)} \leq M 2^{\Psi(n)} .
$$

Therefore we may write the above calculated estimate as:

$$
\begin{aligned}
(* *) \quad\|T(t) z-z\| \leq\left(\left\|T\left(\alpha_{m}\right) z-T\left(\alpha_{\infty}\right) z\right\|+\left\|z-T\left(\alpha_{m}\right) z\right\|\right) M \sum_{i=1}^{n} 2^{\Psi(i)} \\
+M \sum_{i=1}^{n} 2^{\Psi(i)}\left\|T\left(\alpha_{i}\right) z-z\right\|+\left\|T(t) z-T\left(\sum_{i=1}^{n} k_{i} \beta_{i}\right) z\right\|
\end{aligned}
$$

Now consider, together with the uniform equicontinuity assumption for the semigroup (as $m \in \mathbb{N}$ was arbitrary)

$$
\begin{aligned}
\forall b \in \mathbb{N} \forall z \in C_{b} \forall k \in \mathbb{N} \forall L \in \mathbb{N} \forall m & \in \mathbb{N} \forall \alpha_{\infty}, \alpha_{m} \in[0, L] \\
& \left(\left|\alpha_{\infty}-\alpha_{m}\right|<2^{-\omega_{b, L}(k)} \rightarrow\left\|T\left(\alpha_{\infty}\right) z-T\left(\alpha_{m}\right) z\right\|<2^{-k}\right),
\end{aligned}
$$

the convergence assumption

$$
\forall k \in \mathbb{N} \forall m \geq \Phi(k)\left(\left|\alpha_{m}-\alpha_{\infty}\right|<2^{-k}\right)
$$


that combined give

$$
\begin{gathered}
\forall b \in \mathbb{N} \forall z \in C_{b} \forall k \in \mathbb{N} \forall L \in \mathbb{N} \forall \alpha_{\infty}, \alpha_{m} \in[0, L] \forall m \geq \Phi\left(\omega_{b, L}(k)\right) \\
\left\|T\left(\alpha_{m}\right) z-T\left(\alpha_{\infty}\right) z\right\|<2^{-k} .
\end{gathered}
$$

Now, the convergence statement (as already mentioned by [20, Lemma 2] here we have again the same rate of convergence $\Phi$ )

$$
\forall m \in \mathbb{N} \forall n \geq \Phi(m)\left(\left|\sum_{i=1}^{n} k_{i} \beta_{i}-t\right|<2^{-m}\right)
$$

combined with the uniform equicontinuity assumption for the semigroup (notice that for $t \in[0, M]$ if $n \geq \Phi(m)$ by the above we have $\left.\left|\sum_{i=1}^{n} k_{i} \beta_{i}-t\right| \in[0, M+1]\right)$ gives

$$
\begin{gathered}
\forall b \in \mathbb{N} \forall z \in C_{b} \forall k \in \mathbb{N} \forall t \in[0, M] \forall n \geq \Phi\left(\omega_{b, M+1}(k)\right) \\
\left\|T\left(\sum_{i=1}^{n} k_{i} \beta_{i}\right) z-T(t) z\right\|<2^{-k} .
\end{gathered}
$$

Substituting in (**), for a given $j \in \mathbb{N}$ which satisfies

$$
\forall n \in \mathbb{N}\left\|T\left(\alpha_{n}\right) z-z\right\|<2^{-j}
$$

we obtain

$$
\begin{array}{r}
\forall k \in \mathbb{N} \forall t \in[0, M]\|T(t) z-z\| \leq\left(2^{-j}+\left\|z-T\left(\alpha_{\Phi\left(\omega_{b, L}(j)\right)}\right) z\right\|\right) M \sum_{i=1}^{\Phi\left(\omega_{b, M+1}(k)\right)} 2^{\Psi(i)} \\
+M \sum_{i=1}^{\Phi\left(\omega_{b, M+1}(k)\right)} 2^{\Psi(i)}\left\|T\left(\alpha_{i}\right) z-z\right\|+2^{-k}
\end{array}
$$

Because

$$
\begin{aligned}
& \forall k \in \mathbb{N} \exists n \in \mathbb{N}\left(\left\|T\left(\alpha_{n}\right) z-z\right\|<2^{-j} \rightarrow\right. \\
& \left.\left\|z-T\left(\alpha_{\Phi\left(\omega_{b, L}(j)\right)}\right) z\right\|<2^{-j} \wedge \sum_{i=1}^{\Phi\left(\omega_{b, M+1}(k)\right)} 2^{\Psi(i)}\left\|T\left(\alpha_{i}\right) z-z\right\|<2^{-j} \sum_{i=1}^{\Phi\left(\omega_{b, M+1}(k)\right)} 2^{\Psi(i)}\right)
\end{aligned}
$$

we have

$$
n \leq \max \left\{\Phi\left(\omega_{b, M+1}(k)\right), \Phi\left(\omega_{b, L}(j)\right)\right\}
$$


In total, as $M \in \mathbb{N}$ was arbitrary:

$$
\begin{aligned}
& \forall b \in \mathbb{N} \forall z \in C_{b} \forall k \in \mathbb{N} \forall M \in \mathbb{N} \forall t \in[0, M] \forall L \in \mathbb{N} \forall \alpha_{n}, \alpha_{\infty} \in[0, L] \\
& \exists n \leq \max \left\{\Phi\left(\omega_{b, M+1}(k)\right), \Phi\left(\omega_{b, L}(j)\right)\right\}\left(\left\|T\left(\alpha_{n}\right) z-z\right\| \leq 2^{-j} \rightarrow\right. \\
& \|T(t) z-z\|<\left(2^{-j}+2^{-j}\right) M \sum_{i=1}^{\Phi\left(\omega_{b, M+1}(k)\right)} 2^{\Psi(i)}+2^{-j} M \sum_{i=1}^{\Phi\left(\omega_{b, M+1}(k)\right)} 2^{\Psi(i)}+2^{-k} \\
& \left.=3 \cdot 2^{-j} M \sum_{i=1}^{\Phi\left(\omega_{b, M+1}(k)\right)} 2^{\Psi(i)}+2^{-k}\right)
\end{aligned}
$$

Now let the above arbitrary $j \in \mathbb{N}$ be such that for a yet to be determined $k \in \mathbb{N}$,

$$
2^{-j} \leq \frac{2^{-k}}{3 M \sum_{i=1}^{\Phi\left(\omega_{b, M+1}(k)\right)} 2^{\Psi(i)}} .
$$

Choosing

$$
j:=k+\left\lceil\log _{2}\left(3 M \sum_{i=1}^{\Phi\left(\omega_{b, M+1}(k)\right)} 2^{\Psi(i)}\right)\right\rceil
$$

we thus have

$$
\begin{aligned}
& \forall b \in \mathbb{N} \forall z \in C_{b} \forall k \in \mathbb{N} \forall M \in \mathbb{N} \forall t \in[0, M] \forall L \in \mathbb{N} \\
& \exists n \leq \max \left\{\Phi\left(\omega_{b, M+1}(k)\right), \Phi\left(\omega_{b, L}\left(k+\left\lceil\log _{2}\left(3 M \sum_{i=1}^{\Phi\left(\omega_{b, M+1}(k)\right)} 2^{\Psi(i)}\right)\right\rceil\right)\right)\right\} \\
&\left(\left\|T\left(\alpha_{n}\right) z-z\right\| \leq \frac{2^{-k}}{3 M \sum_{i=1}^{\Phi\left(\omega_{b, M+1}(k)\right)} 2^{\Psi(i)}} \rightarrow\|T(t) z-z\|<2^{-k}+2^{-k}\right)
\end{aligned}
$$

ie we have extracted the bounds:

$$
\begin{aligned}
& \tilde{W}:=\max \left\{\Phi\left(\omega_{b, M+1}(k+1)\right), \Phi\left(\omega_{b, L}\left(k+1+\left\lceil\log _{2}\left(3 M \sum_{i=1}^{\Phi\left(\omega_{b, M+1}(k+1)\right)} 2^{\Psi(i)}\right)\right\rceil\right)\right)\right\} \\
& W:=\frac{2^{-(k+1)}}{3 M \sum_{i=1}^{\Phi\left(\omega_{b, M+1}(k+1)\right)} 2^{\Psi(i)}}
\end{aligned}
$$

Lemma 3.5 (Suzuki [20, Lemma 3] ) Let $\alpha, \beta \in \mathbb{R}^{+}$satisfying $\alpha / \beta \notin \mathbb{Q}$. Define sequences $\left\{\alpha_{n}\right\} \in(0, \infty)$ and $\left\{k_{n}\right\} \in \mathbb{N}$ as follows:

- $\alpha_{1}=\max \{\alpha, \beta\}$ 
- $\alpha_{2}=\min \{\alpha, \beta\}$

- $k_{n}=\left[\alpha_{n} / \alpha_{n+1}\right]$ for all $n \in \mathbb{N}$

- $\alpha_{n+2}=\alpha_{n}-k_{n} \alpha_{n+1}$ for all $n \in \mathbb{N}$

Then the following hold:

- $0<\alpha_{n+1}<\alpha_{n}$ for all $n \in \mathbb{N}$

- $k_{n} \in \mathbb{N}$ for all $n \in \mathbb{N}$

- $\alpha_{n} / \alpha_{n+1} \notin \mathbb{Q}$ for all $n \in \mathbb{N}$

- $\left\{\alpha_{n}\right\}$ converges to 0

We show the following:

Lemma 3.6 (Quantitative version of [20, Lemma 3]) Let $2^{-G}<\alpha, \beta \in \mathbb{R}^{+}$for some $G \in \mathbb{N}$ and satisfying $\alpha<\beta$ and $\beta / \alpha \in \mathbb{R} \backslash \mathbb{Q}$ with an effective irrationality measure (with domain restricted to $\mathbb{N} \times \mathbb{N}) f_{\frac{\beta}{\alpha}}$. Define a sequence $\left\{\alpha_{n}\right\} \in(0, \infty)$ as

$$
\alpha_{1}:=\beta, \alpha_{2}:=\alpha, \alpha_{n+2}:=\alpha_{n}-\left[\frac{\alpha_{n}}{\alpha_{n+1}}\right] \alpha_{n+1}
$$

Then:

$$
\forall n \in \mathbb{N} \alpha_{n}>\alpha_{n+1}>0 \text { and } \forall n \in \mathbb{N} \frac{\alpha_{n}}{\alpha_{n+1}} \in \mathbb{R} \backslash \mathbb{Q}
$$

$$
\forall n \in \mathbb{N} \alpha_{n}>2^{-\Psi(n)}
$$

where $\Psi(n)$ is defined simultaneously with $f_{\frac{\alpha_{n}}{\alpha_{n+1}}}: \mathbb{N} \times \mathbb{N} \rightarrow \mathbb{N}$ as follows:

$$
\begin{aligned}
& f_{\frac{\alpha_{1}}{\alpha_{2}}}(p, q):=f_{\frac{\beta}{\alpha}}(p, q) \\
& f_{\frac{\alpha_{n+1}}{\alpha_{n+2}}}(p, q):=\max _{k \leq\lceil\beta\rceil 2^{\Psi(n+1)}}\left\{f_{\frac{\alpha_{n}}{\alpha_{n+1}}}(k p+q, p)\right\}\left\lceil\frac{q}{p}\right\rceil \\
& \Psi(1):=G, \quad \Psi(2):=G, \text { and for } n>2 \\
& \Psi(n):=\sum_{i=2}^{n-2}\left\lceil\log _{2}\left(\max _{l \leq\lceil\beta\rceil 2^{\Psi(i+1)}}\left\{f_{\frac{\alpha_{i}}{\alpha_{i+1}}}(l, 1)\right\}\right)\right\rceil+G \\
& \forall k \in \mathbb{N} \forall n \geq \Phi(k) \alpha_{n}<2^{-k} \text { with } \Phi(k):=\lceil\beta\rceil 2^{k}+2 .
\end{aligned}
$$


Proof The proof of (A) is carried out by induction, it is in fact the proof of Lemma 3.5 that is given in [20] and we present it here as for the next step we will write down a quantitative version of it. By definition $\alpha_{1} / \alpha_{2}=\beta / \alpha \in \mathbb{R} \backslash \mathbb{Q}$ and $\alpha_{1}=\beta>\alpha_{2}=$ $\alpha>0$ so $A(n=1)$ holds. Consider the induction hypothesis:

$(A(j)) \quad$ for some $j \in \mathbb{N} 0<\alpha_{j+1}<\alpha_{j}$ and $\frac{\alpha_{j}}{\alpha_{j+1}} \in \mathbb{R} \backslash \mathbb{Q}$

(Note that then in particular $\left[\alpha_{j} / \alpha_{j+1}\right] \geq 1$.)

By the definition of the sequence we have

$$
\alpha_{j+2}=\left(\frac{\alpha_{j}}{\alpha_{j+1}}-\left[\frac{\alpha_{j}}{\alpha_{j+1}}\right]\right) \alpha_{j+1}
$$

and by the definition of the floor function $[\cdot]$ for any $x \in \mathbb{R}$ we have $x-[x] \in[0,1)$ while here, by $(A(j))$,

therefore

$$
\frac{\alpha_{j}}{\alpha_{j+1}}-\left[\frac{\alpha_{j}}{\alpha_{j+1}}\right]>0 \text { and } \alpha_{j+1}>0
$$

As by definition

$$
0<\alpha_{j+2}<\alpha_{j+1} \text {. }
$$

$$
\frac{\alpha_{j+2}}{\alpha_{j+1}}=\frac{\alpha_{j}}{\alpha_{j+1}}-\left[\frac{\alpha_{j}}{\alpha_{j+1}}\right],
$$

it is $\frac{\alpha_{j+2}}{\alpha_{j+1}} \in \mathbb{R} \backslash \mathbb{Q}$ and thus $\frac{\alpha_{j+1}}{\alpha_{j+2}} \in \mathbb{R} \backslash \mathbb{Q}$. So we have shown $(A(j+1))$ and thus by induction we have shown $(A(n))$, ie $0<\alpha_{n+1}<\alpha_{n}$ and $\frac{\alpha_{n+1}}{\alpha_{n+2}} \in \mathbb{R} \backslash \mathbb{Q}$ for all $n \in \mathbb{N}$. Showing (B) amounts to writing down a quantitative version of (A). Since (A) was shown by induction and the statements on the irrationality of $\frac{\alpha_{n}}{\alpha_{n+1}}$ for all $n \in \mathbb{N}$ and the fact that for all $n \in \mathbb{N} \alpha_{n}>0$ were shown simultaneously, $f_{\frac{\alpha_{n}}{\alpha_{n+1}}}$ is defined recursively and simultaneously with $\Psi(n)$ (the latter is in fact the quantitative information that is of interest here) thus the proof will be carried out again by induction. For $n=1$ by the definition of $\left\{\alpha_{n}\right\}$ we have

$$
f_{\frac{\alpha_{1}}{\alpha_{2}}}(p, q)=f_{\frac{\beta}{\alpha}}(p, q)
$$

where $f_{\frac{\beta}{\alpha}}$ is a function $\mathbb{N} \times \mathbb{N} \rightarrow \mathbb{N}$ so that

$$
\forall p, q \in \mathbb{N}\left|\frac{\beta}{\alpha}-\frac{p}{q}\right| \geq \frac{1}{f_{\frac{\beta}{\alpha}}(p, q)}
$$

ie the effective irrationality measure of $\frac{\beta}{\alpha}$ with the domain restricted to exclude zero (compare with Definition 2.4); moreover, $\alpha_{1}=\beta>2^{-\Psi(1)}$ (respectively $\alpha_{2}=\alpha>$ $\left.2^{-\Psi(2)}\right)$ are clearly fulfilled when

$$
\Psi(1):=G, \Psi(2):=G
$$


as by assumption $\beta>\alpha>2^{-G}$. Consider the following induction hypothesis. Let us assume that, for some $j \in \mathbb{N}$, we have

$(B(j)) \quad \forall p, q \in \mathbb{N}\left|\frac{\alpha_{j}}{\alpha_{j+1}}-\frac{p}{q}\right| \geq \frac{1}{f \frac{\alpha_{j}}{\alpha_{j+1}}(p, q)}$ and $\alpha_{j+1}>2^{-\Psi(j+1)}$.

Using $(B(j))$ we will show $(B(j+1))$.

Notice that for all $p, q \in \mathbb{N}$,

$$
\begin{aligned}
\left|\frac{\alpha_{j+2}}{\alpha_{j+1}}-\frac{p}{q}\right| & =\left|\frac{\alpha_{j}}{\alpha_{j+1}}-\left[\frac{\alpha_{j}}{\alpha_{j+1}}\right]-\frac{p}{q}\right|=\left|\frac{\alpha_{j}}{\alpha_{j+1}}-\left(\frac{\left[\frac{\alpha_{j}}{\alpha_{j+1}}\right] q+p}{q}\right)\right| \\
& \geq \frac{1}{f \frac{\alpha_{j}}{\alpha_{j+1}}\left(\left[\frac{\alpha_{j}}{\alpha_{j+1}}\right] q+p, q\right)} \\
& \geq \frac{1}{\max _{k \leq\lceil\beta\rceil 2^{\Psi(j+1)}\left\{f \frac{\alpha_{j}}{\alpha_{j+1}}(k q+p, q)\right\}}}
\end{aligned}
$$

because $\left[\frac{\alpha_{j}}{\alpha_{j+1}}\right] \leq\left[\frac{\beta}{\alpha_{j+1}}\right] \leq\left[\beta 2^{\Psi(j+1)}\right] \leq \beta 2^{\Psi(j+1)} \leq\lceil\beta\rceil 2^{\Psi(j+1)}$.

Therefore

$$
\begin{aligned}
\forall p, q \in \mathbb{N}\left|\frac{\alpha_{j+1}}{\alpha_{j+2}}-\frac{p}{q}\right| & =\left|\frac{\alpha_{j+1}}{\alpha_{j+2}} \frac{p}{q}\right|\left|\frac{q}{p}-\frac{\alpha_{j+2}}{\alpha_{j+1}}\right| \\
& \geq\left|\frac{\alpha_{j+1}}{\alpha_{j+2}} \frac{p}{q}\right| \frac{1}{\max _{k \leq\lceil\beta\rceil 2^{\Psi(j+1)}}\left\{f \frac{\alpha_{j}}{\alpha_{j+1}}(k p+q, p)\right\}} \\
& \geq\left|\frac{p}{q}\right| \frac{1}{\max _{k \leq\lceil\beta\rceil 2^{\Psi(j+1)}}\left\{f \frac{\alpha_{j}}{\alpha_{j+1}}(k p+q, p)\right\}}
\end{aligned}
$$

(as by (A) $\alpha_{j+1}>\alpha_{j+2}>0$ ) so

$$
f_{\frac{\alpha_{j+1}}{\alpha_{j+2}}}(p, q)=\max _{k \leq\lceil\beta\rceil 2^{\Psi(j+1)}}\left\{f \frac{\alpha_{j}}{\alpha_{j+1}}(k p+q, p)\right\}\left\lceil\frac{q}{p}\right\rceil
$$

indeed gives

$$
\forall p, q \in \mathbb{N}\left|\frac{\alpha_{j+1}}{\alpha_{j+2}}-\frac{p}{q}\right| \geq \frac{1}{f_{\frac{\alpha_{j+1}}{\alpha_{j+2}}}(p, q)} .
$$

We will now show that $\alpha_{j+2}>2^{-\Psi(j+2)}$. To this end, in $(B(j))$ we make the choice (recall that $\left[\alpha_{j} / \alpha_{j+1}\right] \geq 1$ )

$$
p=\left[\frac{\alpha_{j}}{\alpha_{j+1}}\right], q=1
$$


and thus obtain (as by the definition of the floor function [.] for any $x \in \mathbb{R}$ we have $[x] \leq x$ and moreover $\left.\left[\frac{\alpha_{j}}{\alpha_{j+1}}\right] \leq\lceil\beta\rceil 2^{\Psi(j+1)}\right)$ :

$$
\begin{aligned}
\frac{\alpha_{j}}{\alpha_{j+1}}-\left[\frac{\alpha_{j}}{\alpha_{j+1}}\right] & =\left|\frac{\alpha_{j}}{\alpha_{j+1}}-\left[\frac{\alpha_{j}}{\alpha_{j+1}}\right]\right| \geq \frac{1}{f \frac{\alpha_{j}}{\alpha_{j+1}}\left(\left[\frac{\alpha_{j}}{\alpha_{j+1}}\right], 1\right)} \\
& \geq \frac{1}{\max _{l \leq\lceil\beta\rceil 2^{\Psi(j+1)}}\left\{f \frac{\alpha_{j}}{\alpha_{j+1}}(l, 1)\right\}}
\end{aligned}
$$

By recalling that by the definition of $\left\{\alpha_{n}\right\}$ we have

$$
\alpha_{j+2}=\left(\frac{\alpha_{j}}{\alpha_{j+1}}-\left[\frac{\alpha_{j}}{\alpha_{j+1}}\right]\right) \alpha_{j+1}
$$

and by $(B(j))$ we obtain

$$
\alpha_{j+2} \geq \frac{1}{\max _{l \leq\lceil\beta\rceil 2^{\Psi(j+1)}}\left\{f_{\frac{\alpha_{j}}{\alpha_{j+1}}}(l, 1)\right\}} \alpha_{j+1}>\frac{1}{\max _{l \leq\lceil\beta\rceil 2^{\Psi(j+1)}}\left\{f_{\frac{\alpha_{j}}{\alpha_{j+1}}}(l, 1)\right\}} 2^{-\Psi(j+1)} .
$$

Therefore, as by having set

$$
\Psi(n):=\sum_{i=1}^{n-2}\left\lceil\log _{2}\left(\max _{l \leq\lceil\beta\rceil 2^{\Psi(i+1)}}\left\{f_{\frac{\alpha_{i}}{\alpha_{i+1}}}(l, 1)\right\}\right)\right\rceil+\Psi(2)
$$

we have

$$
\Psi(j+2)-\Psi(j+1)=\left\lceil\log _{2}\left(\max _{l \leq\lceil\beta\rceil 2^{\Psi(j+1)}}\left\{f_{\frac{\alpha_{j}}{\alpha_{j+1}}}(l, 1)\right\}\right)\right\rceil .
$$

We have shown by the above that

$$
\alpha_{j+2}>2^{-\Psi(j+2)}
$$

so the proof of $(B(j+1))$ is complete and by induction

$$
\forall n \in \mathbb{N} \alpha_{n}>2^{-\Psi(n)} .
$$

We will now show (C). By (A) $\left\{\alpha_{n}\right\}$ is convergent, and thus Cauchy. We will show that the limit of $\left\{\alpha_{n}\right\}$ is zero, ie that

$$
\forall m \in \mathbb{N} \exists n \forall i, j \geq n\left|\alpha_{i}-\alpha_{j}\right|<2^{-m} \rightarrow \forall k \in \mathbb{N} \exists l \in \mathbb{N} \forall n \geq l \alpha_{n}<2^{-k}
$$

and we will moreover find a computable bound on $l \in \mathbb{N}$. Because by (A) $\left\{\alpha_{n}\right\}$ is decreasing, it is enough to show that

$$
\forall m \in \mathbb{N} \exists n \forall i, j \geq n\left|\alpha_{i}-\alpha_{j}\right|<2^{-m} \rightarrow \forall k \in \mathbb{N} \exists l \in \mathbb{N} \alpha_{l}<2^{-k} .
$$


Note that, in order to derive the quantitative (and effective) information of interest, ie the bound $\Phi$, we will apply proof mining to the entire statement (!), and not to just its conclusion. This is because the premise will be weakened to a metastable Cauchy statement in order to apply Kohlenbach [10, Proposition 2.27].

We claim that the negation of (!) will give a contradiction, that is, we claim that

$$
\begin{gathered}
\forall m \in \mathbb{N} \exists n \in \mathbb{N} \forall i, j \geq n\left|\alpha_{i}-\alpha_{j}\right|<2^{-m} \\
\wedge \exists k \in \mathbb{N} \forall l \in \mathbb{N} \alpha_{l} \geq 2^{-k}
\end{gathered}
$$

will give a contradiction. To show this claim, in (I) above we make the choices ${ }^{2} m:=k$ where $k \in \mathbb{N}$ is as in (II), $i:=n$ and $j:=n+1$, ie:

$$
\exists n\left|\alpha_{n}-\alpha_{n+1}\right|=\alpha_{n}-\alpha_{n+1}<2^{-k}
$$

(as $\left\{\alpha_{n}\right\}$ is decreasing by (A)). The assumption (II) for such a $k$, together with (III) in which $k$ is as in (II) give

$$
2^{-k} \leq \alpha_{n+1}<\alpha_{n}<\alpha_{n+1}+2^{-k}
$$

Dividing the above by $\alpha_{n+1}>0$ we have

so

$$
\frac{2^{-k}}{\alpha_{n+1}} \leq 1<\frac{\alpha_{n}}{\alpha_{n+1}}<\frac{\alpha_{n+1}+2^{-k}}{\alpha_{n+1}}=1+\frac{2^{-k}}{\alpha_{n+1}} \leq 2
$$

which gives

$$
1<\frac{\alpha_{n}}{\alpha_{n+1}}<2
$$

$$
\left[\frac{\alpha_{n}}{\alpha_{n+1}}\right]=1 \text {. }
$$

Now, by the definition of the sequence $\left\{\alpha_{n}\right\}$, substituting the above we obtain

$$
\alpha_{n+2}=\alpha_{n}-\left[\frac{\alpha_{n}}{\alpha_{n+1}}\right] \alpha_{n+1}=\alpha_{n}-\alpha_{n+1}<2^{-k}
$$

and clearly $\alpha_{n+2}<2^{-k}$ gives a contradiction to the assumption (II) for $l:=n+2$.

Thus the bound $\Phi$ on $l$ corresponds to a bound on $n$ shifted by 2. The latter is obtained by applying [10, Proposition 2.27] (also see Remark 2.29 there). Since $\left\{\alpha_{n}\right\} \in(0, \beta] \subseteq[0,\lceil\beta\rceil]$ we obtain

$$
\Phi(k):=\lceil\beta\rceil 2^{k}+2,
$$

\footnotetext{
${ }^{2}$ this amounts to making the choice $g(k):=1$ for $g$ as in Proposition 2.27 in [10].
} 
ie we have shown that

$$
\forall k \in \mathbb{N} \forall n \geq \Phi(k) \alpha_{n}<2^{-k}
$$

with

$$
\Phi(k):=\lceil\beta\rceil 2^{k}+2 .
$$

We now show our main result.

Theorem 3.7 (Quantitative version of [20, Proposition 2]) Let $X$ be a Banach space and let $\{T(t): t \geq 0\}$ be a one-parameter uniformly equicontinuous semigroup of nonexpansive mappings on a subset $C$ of $X$ with a modulus of uniform equicontinuity $\omega$. Let $\alpha, \beta \in \mathbb{R}^{+}$with $2^{-G}<\alpha<\beta$ for some $G \in \mathbb{N}$ and satisfying $\beta / \alpha \in \mathbb{R} \backslash \mathbb{Q}$ with an effective irrationality measure (with domain restricted to $\mathbb{N} \times \mathbb{N}$ ) $f_{\frac{\beta}{\alpha}}$. Then

$$
\begin{aligned}
\forall k \in \mathbb{N} \forall M \in \mathbb{N} \forall b & \in \mathbb{N} \forall z \in C_{b} \\
(\|T(\alpha) z-z\| & \left.\leq \mathcal{X} \wedge\|T(\beta) z-z\| \leq \mathcal{X} \rightarrow \forall t \in[0, M]\|T(t) z-z\|<2^{-k}\right)
\end{aligned}
$$

with

$$
\begin{aligned}
\mathcal{X} & =\mathcal{X}\left(f_{\frac{\beta}{\alpha}},\lceil\beta\rceil, G, b, M, k, \Phi, \Psi, \omega, \tilde{W}\right) \\
& =\frac{\sqrt{5} \frac{2^{-(k+1)}}{6 M \sum_{i=1}^{\Phi\left(\omega_{b}, M+1^{(k+1))}\right.} 2^{\Psi(i)}}}{\left(\left(\frac{1+\sqrt{5}}{2}\right)^{\tilde{W}-1}-\left(\frac{1-\sqrt{5}}{2}\right)^{\tilde{W}-1}\right) \prod_{i=1}^{\tilde{W}-2}\lceil\beta\rceil 2^{\Psi(i+1)}}
\end{aligned}
$$

where

$$
\Psi(1):=G, \Psi(2):=G
$$

and for $n>2$

$$
\Psi(n):=\sum_{i=2}^{n-2}\left\lceil\log _{2}\left(\max _{l \leq\lceil\beta\rceil 2^{\Psi(i+1)}}\left\{f \frac{\alpha_{i}}{\alpha_{i+1}}(l, 1)\right\}\right)\right\rceil+G
$$

with

$$
f_{\frac{\alpha_{1}}{\alpha_{2}}}(p, q):=f_{\frac{\beta}{\alpha}}(p, q)
$$

and

$$
f_{\frac{\alpha_{n+1}}{\alpha_{n+2}}}(p, q):=\max _{k \leq\lceil\beta\rceil 2^{\Psi(n+1)}}\left\{f_{\frac{\alpha_{n}}{\alpha_{n+1}}}(k p+q, p)\right\}\left\lceil\frac{q}{p}\right\rceil,
$$

where $\left\{\alpha_{n}\right\}$ is a sequence defined by $\alpha_{1}:=\beta, \alpha_{2}:=\alpha, \alpha_{n+2}:=\alpha_{n}-\left[\frac{\alpha_{n}}{\alpha_{n+1}}\right] \alpha_{n+1}$,

$$
\Phi(k):=\lceil\beta\rceil 2^{k}+2
$$


and

$$
\begin{aligned}
\tilde{W} & =\tilde{W}(k, b, M,\lceil\beta\rceil, \Phi, \Psi, \omega) \\
& =\max \left\{\Phi\left(\omega_{b, M+1}(k+1)\right), \Phi\left(\omega_{b,\lceil\beta\rceil}\left(k+1+\left\lceil\log _{2}\left(3 M \sum_{i=1}^{\Phi\left(\omega_{b, M+1}(k+1)\right)} 2^{\Psi(i)}\right)\right\rceil\right)\right)\right\} .
\end{aligned}
$$

Proof Define a sequence $\left\{\alpha_{n}\right\} \in(0, \infty)$ as in Lemma 3.6. For convenience set

$$
k_{n}:=\left[\alpha_{n} / \alpha_{n+1}\right] .
$$

We have:

$$
\begin{aligned}
\left\|T\left(\alpha_{n+2}\right) z-z\right\| & =\left\|T\left(\alpha_{n+2}\right) z-z+T\left(\alpha_{n}\right) z-T\left(\alpha_{n}\right) z\right\| \\
& \leq\left\|T\left(\alpha_{n+2}\right) z-T\left(\alpha_{n}\right) z\right\|+\left\|T\left(\alpha_{n}\right) z-z\right\| \\
& =\left\|T\left(\alpha_{n+2}\right) z-T\left(\alpha_{n+2}+k_{n} \alpha_{n+1}\right) z\right\|+\left\|T\left(\alpha_{n}\right) z-z\right\| \\
& =\left\|T\left(\alpha_{n+2}\right) z-T\left(\alpha_{n+2}\right) T\left(k_{n} \alpha_{n+1}\right) z\right\|+\left\|T\left(\alpha_{n}\right) z-z\right\| \\
& \leq\left\|z-T\left(k_{n} \alpha_{n+1}\right) z\right\|+\left\|T\left(\alpha_{n}\right) z-z\right\| \\
& =\left\|z-T^{k_{n}}\left(\alpha_{n+1}\right) z\right\|+\left\|T\left(\alpha_{n}\right) z-z\right\| \\
& =\left\|z-T^{k_{n}}\left(\alpha_{n+1}\right) z+T\left(\alpha_{n+1}\right) z-T\left(\alpha_{n+1}\right) z\right\|+\left\|T\left(\alpha_{n}\right) z-z\right\| \\
& \leq\left\|T^{k_{n}}\left(\alpha_{n+1}\right) z-T\left(\alpha_{n+1}\right) z\right\|+\left\|T\left(\alpha_{n+1}\right) z-z\right\|+\left\|T\left(\alpha_{n}\right) z-z\right\| \\
& \leq\left\|T^{k_{n}-1}\left(\alpha_{n+1}\right) z-z\right\|+\left\|T\left(\alpha_{n+1}\right) z-z\right\|+\left\|T\left(\alpha_{n}\right) z-z\right\| \\
& \quad \vdots \\
& \leq k_{n}\left\|T\left(\alpha_{n+1}\right) z-z\right\|+\left\|T\left(\alpha_{n}\right) z-z\right\|
\end{aligned}
$$

We have therefore shown that

$$
\begin{aligned}
\left\|T\left(\alpha_{n+2}\right) z-z\right\| & \leq k_{n}\left\|T\left(\alpha_{n+1}\right) z-z\right\|+\left\|T\left(\alpha_{n}\right) z-z\right\| \\
& \leq k_{n}\left(\left\|T\left(\alpha_{n+1}\right) z-z\right\|+\left\|T\left(\alpha_{n}\right) z-z\right\|\right)
\end{aligned}
$$

(because by Lemma $3.60<\alpha_{n+1}<\alpha_{n}$ for all $n \in \mathbb{N}$, thus $k_{n}=\left[\alpha_{n} / \alpha_{n+1}\right] \geq 1$ for all $n \in \mathbb{N}$ ).

Now let $b \in \mathbb{N}$ and $z \in C_{b}$ such that

$$
\left\|T\left(\alpha_{1}\right) z-z\right\|=\|T(\beta) z-z\| \leq \delta \wedge\left\|T\left(\alpha_{2}\right) z-z\right\|=\|T(\alpha) z-z\| \leq \delta
$$

for some $\delta>0$. Let us consider the sequence

$$
\left\{\left\|T\left(\alpha_{n}\right) z-z\right\|\right\}_{n \in \mathbb{N}}
$$


satisfying

$$
\left\|T\left(\alpha_{n+2}\right) z-z\right\| \leq k_{n}\left(\left\|T\left(\alpha_{n+1}\right) z-z\right\|+\left\|T\left(\alpha_{n}\right) z-z\right\|\right)
$$

and

$$
\left\|T\left(\alpha_{1}\right) z-z\right\|=\|T(\beta) z-z\| \leq \delta \text { and }\left\|T\left(\alpha_{2}\right) z-z\right\|=\|T(\alpha) z-z\| \leq \delta
$$

for some $\delta>0$. We will estimate the $n$th term as follows. Observing the form of the first terms:

$$
\begin{aligned}
\left\|T\left(\alpha_{3}\right) z-z\right\| & \leq k_{1} 2 \delta \\
\left\|T\left(\alpha_{4}\right) z-z\right\| & \leq k_{2}\left(k_{1} 2 \delta+\delta\right)=\delta\left(k_{2}+2 k_{1} k_{2}\right) \\
\left\|T\left(\alpha_{5}\right) z-z\right\| & \leq k_{3}\left(\delta\left(k_{2}+2 k_{1} k_{2}\right)+k_{1} 2 \delta\right)=\delta\left(k_{3} k_{2}+2 k_{1} k_{2} k_{3}+2 k_{1} k_{3}\right), \\
\left\|T\left(\alpha_{6}\right) z-z\right\| & \leq k_{4}\left(\delta\left(k_{3} k_{2}+2 k_{1} k_{2} k_{3}+2 k_{1} k_{3}\right)+\delta\left(k_{2}+2 k_{1} k_{2}\right)\right) \\
& =\delta\left(k_{4} k_{3} k_{2}+2 k_{4} k_{3} k_{2} k_{1}+2 k_{4} k_{1} k_{3}+k_{4} k_{2}+2 k_{4} k_{2} k_{1}\right) \\
& \left(\leq 2 \delta\left(k_{4} k_{3} k_{2}+k_{4} k_{3} k_{2} k_{1}+k_{4} k_{1} k_{3}+k_{4} k_{2}+k_{4} k_{2} k_{1}\right)\right)
\end{aligned}
$$

Now note that because, as mentioned above, for all $n \in \mathbb{N}, k_{n} \geq 1$, for $n \leq m$ we have

$$
\prod_{i=1}^{n} k_{i} \leq \prod_{i=1}^{m} k_{i}
$$

Moreover, note that the number of summands in the respective bound of each term of the above sequence (where each summand is a product of $k_{i} \mathrm{~s}$ ) clearly follows the Fibonacci sequence, ie:

\begin{tabular}{cc} 
number of summands of products of $k_{i} \mathrm{~s}$ \\
\hline$\left\|T\left(\alpha_{3}\right) z-z\right\|$ & 1 \\
$\left\|T\left(\alpha_{4}\right) z-z\right\|$ & 2 \\
$\left\|T\left(\alpha_{5}\right) z-z\right\|$ & 3 \\
$\left\|T\left(\alpha_{6}\right) z-z\right\|$ & 5 \\
$\vdots$ & $\vdots$
\end{tabular}

as each term approximation involves the sum of the two previous term approximations. In particular, the $n$th term of the sequence $\left\{\left\|T\left(\alpha_{n}\right) z-z\right\|\right\}_{n \in \mathbb{N}}$ has a factor which is the $n-1$ th Fibonacci number. The $n$th Fibonacci number is given by the well-known Binet's formula:

$$
F_{n}=\frac{\left(\frac{1+\sqrt{5}}{2}\right)^{n}-\left(\frac{1-\sqrt{5}}{2}\right)^{n}}{\sqrt{5}}
$$


Hence, for the $n$th term of the sequence $\left\{\left\|T\left(\alpha_{n}\right) z-z\right\|\right\}_{n \in \mathbb{N}}$ we have:

$$
\left\|T\left(\alpha_{n}\right) z-z\right\| \leq \frac{\left(\frac{1+\sqrt{5}}{2}\right)^{n-1}-\left(\frac{1-\sqrt{5}}{2}\right)^{n-1}}{\sqrt{5}} 2 \prod_{i=1}^{n-2} k_{i}
$$

Moreover, note that, for each $i \in \mathbb{N}$ :

$$
k_{i}=\left[\alpha_{i} / \alpha_{i+1}\right] \leq\left[\alpha_{1} / \alpha_{i+1}\right]=\left[\beta / \alpha_{i+1}\right] \leq\left[\beta 2^{\Psi(i+1)}\right] \leq \beta 2^{\Psi(i+1)} \leq\lceil\beta\rceil 2^{\Psi(i+1)}
$$

Therefore we may write:

$$
\left\|T\left(\alpha_{n}\right) z-z\right\| \leq \frac{\left(\frac{1+\sqrt{5}}{2}\right)^{n-1}-\left(\frac{1-\sqrt{5}}{2}\right)^{n-1}}{\sqrt{5}} 2 \prod_{i=1}^{n-2}\lceil\beta\rceil 2^{\Psi(i+1)}
$$

Thus, for

$$
n:=\tilde{W}
$$

with $\tilde{W}$ as it was previously obtained in our Theorem 3.4 with $L:=\lceil\beta\rceil$, as the above obtained bound on $\left\|T\left(\alpha_{n}\right) z-z\right\|$ is nondecreasing on $n$ we have that

$$
\begin{aligned}
\|T(\alpha) z-z\| \leq \delta \wedge\|T(\beta) z-z\| \leq \delta \rightarrow \\
\forall n \leq \tilde{W}\left\|T\left(\alpha_{n}\right) z-z\right\| \leq \frac{\left(\frac{1+\sqrt{5}}{2}\right)^{\tilde{W}-1}-\left(\frac{1-\sqrt{5}}{2}\right)^{\tilde{W}-1}}{\sqrt{5}} 2 \delta \prod_{i=1}^{\tilde{W}-2}\lceil\beta\rceil 2^{\Psi(i+1)} .
\end{aligned}
$$

By choosing $\delta>0$ to be such that

$$
\frac{\left(\frac{1+\sqrt{5}}{2}\right)^{\tilde{W}-1}-\left(\frac{1-\sqrt{5}}{2}\right)^{\tilde{W}-1}}{\sqrt{5}} 2 \delta \prod_{i=1}^{\tilde{W}-2}\lceil\beta\rceil 2^{\Psi(i+1)} \leq W
$$

where $W$ is the bound extracted in Theorem 3.4, ie by choosing

$$
\delta \leq \frac{\sqrt{5} \frac{2^{-(k+1)}}{6 M \sum_{i=1}^{\Phi\left(\omega_{b, M+1}(k+1)\right)} 2^{\Psi(i)}}}{\left(\left(\frac{1+\sqrt{5}}{2}\right)^{\tilde{W}-1}-\left(\frac{1-\sqrt{5}}{2}\right)^{\tilde{W}-1}\right) \prod_{i=1}^{\tilde{W}-2}\lceil\beta\rceil 2^{\Psi(i+1)}}
$$

the premise of Theorem 3.4 is now fulfilled, and therefore by Theorem 3.4 we obtain that

$$
\begin{aligned}
\forall b \in \mathbb{N} \forall z \in C_{b} \forall k & \in \mathbb{N} \forall M \in \mathbb{N} \forall t \in[0, M] \\
& \left(\|T(\alpha) z-z\| \leq \mathcal{X} \wedge\|T(\beta) z-z\| \leq \mathcal{X} \rightarrow\|T(t) z-z\|<2^{-k}\right)
\end{aligned}
$$


with

$$
\mathcal{X}:=\frac{\sqrt{5} \frac{2^{-(k+1)}}{6 M \sum_{i=1}^{\Phi\left(\omega_{b, M+1}(k+1)\right)} 2^{\Psi(i)}}}{\left(\left(\frac{1+\sqrt{5}}{2}\right)^{\tilde{W}-1}-\left(\frac{1-\sqrt{5}}{2}\right)^{\tilde{W}-1}\right) \prod_{i=1}^{\tilde{W}-2}\lceil\beta\rceil 2^{\Psi(i+1)}}
$$

where $\Phi, \Psi$ for the particular sequence $\left\{\alpha_{n}\right\}$ are as extracted in Lemma 3.6.

Remark 1 Corollary to the proof. It would be possible to remove the equicontinuity assumption for the semigroup $\{T(t): t \geq 0\}$. Then the modulus of continuity of $\{T(t): t \geq 0\}$, and thus also the final bound, would depend on $z \in C$ instead of the input $b \in \mathbb{N}$, so that $C_{b}:=\{z \in C: z \leq\|b\|\}$. That would, strictly speaking, constitute a direct quantitative version of Suzuki's result Theorem 3.1. However, omitting our supplementary equicontinuity assumption would have the following disadvantages:

- Clearly the result would be less uniform.

- It would not be possible to derive the corollary on asymptotic regularity that we will derive in the end of this section.

\subsection{Comparison with bound obtained in [13]}

We can compare our result here with the recent result by Kohlenbach and the author in [13], which was obtained by proof mining on a proof of a relevant statement again by Suzuki in [21]. In particular, [21, Theorem 1] states that the set of common fixed points of $\{T(t): t \geq 0\}$ is the set of fixed points of the mapping $\lambda T(\alpha)+(1-\lambda) T(\beta)$, where $\alpha, \beta>0, \alpha / \beta \in \mathbb{R} \backslash \mathbb{Q}$ and $\lambda \in(0,1)$. Clearly if $q \in F(T(\alpha)) \cap F(T(\beta))$ then $q \in F(\lambda T(\alpha)+(1-\lambda) T(\beta))$ for any $\lambda \in(0,1)$, as assuming that for some $q \in C$ we have

$$
T(\alpha) q=q \wedge T(\beta) q=q
$$

we obtain

$$
(\lambda T(\alpha)+(1-\lambda) T(\beta)) q=\lambda T(\alpha) q+(1-\lambda) T(\beta) q=\lambda q+(1-\lambda) q=q .
$$

However, the converse does not hold, so Theorem 1 in [21] is a generalization of [20, Proposition 2].

In [13] a quantitative version of [21, Theorem 1] was given:

Theorem 3.8 (Kohlenbach and Koutsoukou-Argyraki [13, Theorem 2], quantitative version of [21, Theorem 1]) Let $\{T(t): t \geq 0\}$ be a one-parameter nonexpansive 
semigroup on $C \subseteq X$ for some Banach space $X$. Let $\alpha, \beta \in \mathbb{R}^{+}$with $0<\alpha<\beta$. Let $\gamma:=\alpha / \beta \in \mathbb{R}^{+} \backslash \mathbb{Q}^{+}$with an effective irrationality measure $f_{\gamma}$. Let

$$
\lambda T(\alpha)+(1-\lambda) T(\beta)
$$

be a mapping of $C$ into $X$ with $\lambda \in(0,1)$. Moreover assume that $\{T(t): t \geq 0\}$ is uniformly equicontinuous with a modulus of uniform equicontinuity $\omega$. Let $\Lambda \in \mathbb{N}$ be such that $1 / \Lambda \leq \lambda, 1-\lambda, N \in \mathbb{N}$ so that $\beta \geq 1 / N, \mathbb{N} \ni D \geq \beta$. Then

$$
\begin{aligned}
& \forall b \in \mathbb{N} \forall M \in \mathbb{N} \forall z \in C_{b} \forall m \in \mathbb{N} \\
& \quad\left(\|(\lambda T(\alpha)+(1-\lambda) T(\beta)) z-z\| \leq \Psi \rightarrow \forall t \in[0, M]\|T(t) z-z\|<2^{-m}\right)
\end{aligned}
$$

with

$$
\Psi=\Psi\left(m, M, N, \Lambda, D, b, f_{\gamma}, \omega\right)=\frac{2^{-m}}{8\left(\sum_{i=1}^{\phi\left(k, f_{\gamma}\right)-1} \Lambda^{i}+1\right)(1+M N)}
$$

where $k:=D 2^{\omega_{D, b}\left(3+\left[\log _{2}(1+M N)\right]+m\right)+1} \in \mathbb{N}$, and

$$
\phi(k, f):=\max \{2 f(i-j)+6,0 \leq j<i \leq k+1\} \in \mathbb{N} .
$$

Let us assume that we have $z \in C_{b}$ so that, for some $\delta>0$,

$$
\|T(\alpha) z-z\| \leq \delta \wedge\|T(\beta) z-z\| \leq \delta .
$$

Then

$$
\begin{aligned}
\|(\lambda T(\alpha)+(1-\lambda) T(\beta)) z-z\| & =\|(\lambda T(\alpha)+(1-\lambda) T(\beta)) z-z+\lambda z-\lambda z\| \\
& \leq \lambda\|T(\alpha) z-z\|+(1-\lambda)\|T(\beta) z-z\|<\delta .
\end{aligned}
$$

In the above theorem let us make the choice $\lambda:=\frac{1}{2} \in(0,1)$ and $\Lambda:=2$. Therefore the bound could be stated as:

$$
\begin{aligned}
& \forall b \in \mathbb{N} \forall z \in C_{b} \forall M \in \mathbb{N} \forall m \in \mathbb{N} \\
& \quad\left((\|T(\alpha) z-z\| \leq \Psi \wedge\|T(\beta) z-z\| \leq \Psi) \rightarrow \forall t \in[0, M]\|T(t) z-z\|<2^{-m}\right)
\end{aligned}
$$

with

$$
\Psi=\Psi\left(m, M, N, D, b, f_{\gamma}, \omega\right)=\frac{2^{-m}}{8\left(\sum_{i=1}^{\phi\left(k, f_{\gamma}\right)-1} 2^{i}+1\right)(1+M N)}
$$

where $k \in \mathbb{N}$ and $\phi(k, f) \in \mathbb{N}$ are as before.

Comparing the bound $\Psi$ that would follow from the above result shown in [13] to the bound $\mathcal{X}$ obtained in Theorem 3.7 here we make the interesting observation that proof mining on Suzuki's two completely different proofs of essentially the same statement gave us a completely different result. We cannot a priori determine in general 
which bound gives a result that is numerically better, this may differ given different examples of semigroups and/or different choice of input data. Note that both proofs by Suzuki (in [21] and [20] respectively), although completely different to each other, used an irrationality assumption on the ratio of $\alpha$ and $\beta$, thus both the quantitative analyses presented for the first and second approach made use of the notion of effective irrationality measure for an irrational number. (In [13] the effective irrationality measure was taken to depend only on one variable for reasons of simplicity; see the discussion in [13]).

\section{Asymptotic Regularity}

Finally, under the assumption that the Banach space $X$ is moreover uniformly convex, we will now give a corollary to Theorem 3.7 using a result by Khan and Kohlenbach in [5] on the asymptotic regularity of the semigroup $\{T(t): t \geq 0\}$ with respect to a classical iteration schema introduced by Kuhfittig [18] in 1981.

Definition 4.1 (Kuhfittig [18]) Let $C$ be a nonempty convex subset of a Banach space $X$ and let $\left\{T_{i}: 1 \leq i \leq k\right\}$ be a finite family of nonexpansive self-mappings.

Let $U_{0}:=I$ where $I$ denotes the identity mapping. Let $\lambda \in(0,1)$. Consider the mappings:

$$
\begin{gathered}
U_{1}=(1-\lambda) I+\lambda T_{1} U_{0} \\
U_{2}=(1-\lambda) I+\lambda T_{2} U_{1} \\
\vdots \\
U_{k}=(1-\lambda) I+\lambda T_{k} U_{k-1}
\end{gathered}
$$

Define

$$
x_{0} \in C, x_{n+1}:=(1-\lambda) x_{n}+\lambda T_{k} U_{k-1} x_{n}, n \geq 0 .
$$

We recall that:

Definition 4.2 (Clarkson [1], also see Kohlenbach [8]) A Banach space $X$ is called uniformly convex if

$$
\begin{aligned}
& \forall \epsilon \in(0,2] \exists \delta \in(0,1] \forall x, y \in X \\
& \qquad\left(\|x\|,\|y\| \leq 1 \wedge\|x-y\| \geq \epsilon \rightarrow\left\|\frac{1}{2}(x+y)\right\| \leq 1-\delta\right) .
\end{aligned}
$$

A mapping $\eta:(0,2] \rightarrow(0,1]$ giving such a $\delta:=\eta(\epsilon)$ is called a modulus of uniform convexity. 
For example, as a modulus of uniform convexity one may consider Clarkson's modulus of convexity (see [1]) defined for any Banach space $X$ as the function $\eta_{X}:(0,2] \rightarrow(0,1]$ given by

$$
\eta_{X}(\epsilon):=\inf \left\{1-\left\|\frac{x+y}{2}\right\|:\|x\| \leq 1,\|y\| \leq 1,\|x-y\| \geq \epsilon\right\} .
$$

Moreover, we recall:

Definition 4.3 (Krasnoselskii [15], also see Kohlenbach [10]) Let $C$ be a convex subset of a Banach space $X$ and let $T: C \rightarrow C$ nonexpansive. The sequence

$$
x_{n+1}:=\frac{1}{2} x_{n}+\frac{1}{2} T x_{n}
$$

is called the Krasnoselskii iteration of $T$ starting at $x_{0}$.

If

$$
\left\|x_{n}-T x_{n}\right\| \stackrel{n \rightarrow \infty}{\rightarrow} 0
$$

for all $x_{0} \in C$, where $\left\{x_{n}\right\}$ is a given iteration starting at $x_{0}$, then $T$, or more precisely

$$
T_{1 / 2}:=\frac{1}{2}(I+T),
$$

is called asymptotically regular. A rate of convergence for the above convergence is called a rate of asymptotic regularity for $T$.

In the following we will refer to convergence results of the above form also for different iterations $\left\{x_{n}\right\}$ as asymptotic regularity results.

By proof mining on the proof of a theorem by Kuhfittig (implicit) in [18] (also see Theorem 1.2 in [5]), Khan and Kohlenbach showed in [5] the following theorem which is a quantitative version of Kuhfittig's theorem. Note that in [5] Theorem 4.4 is actually shown in the more general context of $U C W$-hyperbolic spaces ${ }^{3}$, but here we state it adapted to the special case of Banach spaces:

Theorem 4.4 (Khan and Kohlenbach [5, Theorem 3.2]) Let $C$ be a nonempty convex subset of a uniformly convex Banach space $X$ with a modulus of uniform convexity $\eta$ and let $\left\{T_{i}: 1 \leq i \leq k\right\}$ be a finite family of nonexpansive self-mappings of $C$ with $\bigcap_{i=1}^{k} F\left(T_{i}\right) \neq \emptyset$. Let $p \in \bigcap_{i=1}^{k} F\left(T_{i}\right)$ and $D>0$ such that $\left\|x_{0}-p\right\| \leq D$ for some

\footnotetext{
${ }^{3} W$-hyperbolic spaces were originally introduced by Kohlenbach in [9] and uniformly convex $W$-hyperbolic spaces by Leuştean in [19].
} 
$x_{0} \in C$. Then for the sequence $\left\{x_{n}\right\}$ generated by the iteration schema of Definition 4.1, we have, for all $1 \leq i \leq k$

$$
\forall \epsilon \in(0,2] \forall n \geq \Theta_{i}(D, \epsilon, N, \eta) \quad\left(\left\|T_{i} x_{n}-x_{n}\right\| \leq \epsilon\right)
$$

with a rate of asymptotic regularity

$$
\Theta_{i}:=\theta\left(\hat{\eta}^{(k-i+\min (1, k-1))}\left(\frac{\epsilon}{2}\right)\right),
$$

where $N \in \mathbb{N}$ is such that $\frac{1}{N} \leq \lambda(1-\lambda)$,

$$
\theta(\epsilon):=\left\lceil\frac{D}{\hat{\eta}(\epsilon)}\right\rceil,
$$

and

$$
\hat{\eta}(\epsilon):=\frac{1}{N} \eta\left(\frac{\epsilon}{D+1}\right) \epsilon
$$

Remark 2 In the case where the Banach space has a modulus of convexity $\eta$ that can be written as $\eta(\epsilon)=\epsilon \tilde{\eta}(\epsilon)$ where $\tilde{\eta}(\epsilon)$ is increasing, (for instance, in the case of the Banach spaces $L_{p}$, that, for $p \geq 2$ have an asymptotically optimal modulus of convexity $\frac{\epsilon^{p}}{p^{2}}$, see Hanner [4], also Kohlenbach [8] and [7] ) then $\eta$ can be replaced with $\tilde{\eta}$ in the bound (see [5, Remark 3.3]).

We show the following corollary to Theorem 3.7 by making use of the above Theorem 4.4.

Corollary 4.5 Let $C$ be a nonempty convex subset of a uniformly convex Banach space $X$ with a modulus of uniform convexity $\eta$ and let $\{T(t): t \geq 0\}$ be a one-parameter uniformly equicontinuous semigroup of nonexpansive mappings on $C$ with a modulus of uniform equicontinuity $\omega$. Let $\alpha, \beta \in \mathbb{R}^{+}$with $2^{-G}<\alpha<\beta$ for some $G \in \mathbb{N}$ and satisfying $\beta / \alpha \in \mathbb{R} \backslash \mathbb{Q}$ with effective irrationality measure (with domain restricted to $\mathbb{N} \times \mathbb{N}) f_{\underline{\beta}}$ and let $F(T(\alpha)) \cap F(T(\beta)) \neq \emptyset$. Let $p \in F(T(\alpha)) \cap F(T(\beta))$ and let $D>0$ such that $\left\|x_{0}-p\right\| \leq D$ for some $x_{0} \in C$. Then for the sequence $\left\{x_{n}\right\}$ generated by the iteration schema of Definition 4.1, we have

$$
\forall k \in \mathbb{N} \forall M \in \mathbb{N} \forall b \in \mathbb{N} \forall n \geq \tilde{\Theta} \forall x_{n} \in C_{b} \forall t \in[0, M]\left\|T(t) x_{n}-x_{n}\right\| \leq 2^{-k}
$$

with a rate of asymptotic regularity

$$
\tilde{\Theta}:=\max _{i=1,2}\left\{\Theta_{i}\right\},
$$


where

$$
\begin{gathered}
\Theta_{i}=\Theta_{i}\left(D, N, \eta, f_{\frac{\beta}{\alpha}}, G,\lceil\beta\rceil, \omega, b, M, k\right):=\theta\left(\hat{\eta}^{(3-i))}\left(\frac{\mathcal{X}}{2}\right)\right), \\
\theta(\epsilon):=\left\lceil\frac{D}{\hat{\eta}(\epsilon)}\right\rceil, \\
\hat{\eta}(\epsilon):=\frac{1}{N} \eta\left(\frac{\epsilon}{D+1}\right) \epsilon,
\end{gathered}
$$

$N \in \mathbb{N}$ is such that $\frac{1}{N} \leq \lambda(1-\lambda)$, and

$$
\begin{aligned}
\mathcal{X} & =\mathcal{X}\left(f_{\frac{\beta}{\alpha}}, G,\lceil\beta\rceil, \tilde{W}, \Psi, \Phi, \omega, b, M, k\right) \\
& =\frac{\sqrt{5} \frac{2^{-(k+1)}}{6 M \sum_{i=1}^{\Phi\left(\omega_{b, M+1}(k+1)\right)} 2^{\Psi(i)}}}{\left(\left(\frac{1+\sqrt{5}}{2}\right)^{\tilde{W}-1}-\left(\frac{1-\sqrt{5}}{2}\right)^{\tilde{W}-1}\right) \prod_{i=1}^{\tilde{W}-2}\lceil\beta\rceil 2^{\Psi(i+1)}}
\end{aligned}
$$

where

$$
\Psi(1):=G, \Psi(2):=G
$$

and for $n>2$

$$
\Psi(n):=\sum_{i=2}^{n-2}\left\lceil\log _{2}\left(\max _{l \leq\lceil\beta\rceil 2^{\Psi(i+1)}}\left\{f_{\frac{\alpha_{i}}{\alpha_{i+1}}}(l, 1)\right\}\right)\right\rceil+G
$$

with

$$
f_{\frac{\alpha_{1}}{\alpha_{2}}}(p, q):=f_{\frac{\beta}{\alpha}}(p, q)
$$

and

$$
f_{\frac{\alpha_{n+1}}{\alpha_{n+2}}}(p, q):=\max _{k \leq\lceil\beta\rceil 2^{\Psi(n+1)}}\left\{f_{\frac{\alpha_{n}}{\alpha_{n+1}}}(k p+q, p)\right\}\left\lceil\frac{q}{p}\right\rceil,
$$

where $\left\{\alpha_{n}\right\}$ is a sequence defined by $\alpha_{1}:=\beta, \alpha_{2}:=\alpha$ and $\alpha_{n+2}:=\alpha_{n}-\left[\frac{\alpha_{n}}{\alpha_{n+1}}\right] \alpha_{n+1}$,

$$
\begin{aligned}
\tilde{W} & =\tilde{W}(k, b, M,\lceil\beta\rceil, \Phi, \Psi, \omega) \\
& =\max \left\{\Phi\left(\omega_{b, M+1}(k+1)\right), \Phi\left(\omega_{b,\lceil\beta\rceil}\left(k+1+\left\lceil\log _{2}\left(3 M \sum_{i=1}^{\Phi\left(\omega_{b, M+1}(k+1)\right)} 2^{\Psi(i)}\right)\right\rceil\right)\right)\right\}
\end{aligned}
$$

and

$$
\Phi(k):=\lceil\beta\rceil 2^{k}+2 .
$$

Proof By Theorem 4.4, for $k=2$ and setting $T_{1}:=T\left(\alpha_{1}\right)=T(\alpha), T_{2}:=T\left(\alpha_{2}\right)=$ $T(\beta)$ we have

with

$$
\begin{aligned}
& \forall \epsilon \in(0,2] \forall n \geq \Theta_{1}(D, \epsilon, N, \eta) \quad\left(\left\|T(\alpha) x_{n}-x_{n}\right\| \leq \epsilon\right), \\
& \forall \epsilon \in(0,2] \forall n \geq \Theta_{2}(D, \epsilon, N, \eta) \quad\left(\left\|T(\beta) x_{n}-x_{n}\right\| \leq \epsilon\right)
\end{aligned}
$$

$$
\Theta_{1}:=\theta\left(\hat{\eta}^{(2)}\left(\frac{\epsilon}{2}\right)\right), \Theta_{2}:=\theta\left(\hat{\eta}\left(\frac{\epsilon}{2}\right)\right)
$$


where $N \in \mathbb{N}$ is such that $\frac{1}{N} \leq \lambda(1-\lambda)$,

and

$$
\begin{gathered}
\theta(\epsilon):=\left\lceil\frac{D}{\hat{\eta}(\epsilon)}\right\rceil, \\
\hat{\eta}(\epsilon):=\frac{1}{N} \eta\left(\frac{\epsilon}{D+1}\right) \epsilon .
\end{gathered}
$$

We may therefore write

$$
\forall \epsilon \in(0,2] \forall n \geq \max _{i=1,2}\left\{\Theta_{i}(D, \epsilon, N, \eta)\right\} \quad\left(\left\|T(\alpha) x_{n}-x_{n}\right\| \leq \epsilon \wedge\left\|T(\beta) x_{n}-x_{n}\right\| \leq \epsilon\right) .
$$

By setting $\epsilon:=\mathcal{X} \in(0,2]$ in the above, where $\mathcal{X}$ is as in Theorem 3.7, the premise of Theorem 3.7 is fulfilled, and we thus directly obtain:

$$
\begin{aligned}
\forall k \in \mathbb{N} \forall M \in \mathbb{N} \forall b \in \mathbb{N} \forall n \geq \max _{i=1,2}\left\{\Theta_{i}(D, \mathcal{X}, N, \eta)\right\} \\
\qquad x_{n} \in C_{b} \forall t \in[0, M]\left\|T(t) x_{n}-x_{n}\right\| \leq 2^{-k}
\end{aligned}
$$

where $\mathcal{X}=\mathcal{X}\left(f_{\frac{\beta}{\alpha}}, G,\lceil\beta\rceil, \tilde{W}, b, M, k, \Phi, \Psi, \omega\right)$ is as in Theorem 3.7.

\section{Acknowledgements}

The author is most grateful to her PhD supervisor Prof. Dr. Ulrich Kohlenbach for several suggestions and corrections that significantly improved an earlier version of this paper. This paper was written as a part of the $\mathrm{PhD}$ work of the author [14] at the Department of Mathematics, Technische Universität Darmstadt, Germany and the author was supported by the IRTG 1529 .

\section{References}

[1] J A Clarkson, Uniformly convex spaces, Trans. Amer. Math. Soc. 40 (1936) 396-414; https://doi.org/10.1090/S0002-9947-1936-1501880-4

[2] MG Crandall, TM Liggett, Generation of semigroups of nonlinear transformations on general Banach spaces, Amer. J. Math. Vol. 93 (1971) 265-298; https://www.jstor.org/stable/2373376 https://doi.org/10.2307/2373376

[3] K Gödel: Über eine bisher noch nicht benützte Erweiterung des finiten Standpunktes, Dialectica Vol. 12 (1958) 280-287; https://doi.org/10.1111/j.1746-8361.1958.tb01464.x

[4] O Hanner: On the uniform convexity of Lp and lp, Ark. Mat. 3 (1956) 239-244; https://doi.org/10.1007/BF02589410 
[5] MA A Khan, U Kohlenbach, Bounds on Kuhfittig's iteration schema in uniformly convex hyperbolic spaces, J. Math. Anal. Appl. 403 (2013) 633-642; https://doi.org/10.1016/j.jmaa.2013.02.058

[6] U Kohlenbach: Theorie der majorisierbaren und stetigen Funktionale und ihre Anwendung bei der Extraktion von Schranken aus inkonstruktiven Beweisen: Effektive Eindeutigkeitsmodule bei besten Approximationen aus ineffektiven Beweisen $\mathrm{PhD}$ Thesis, Frankfurt am Main, xxii+278pp. (1990)

[7] U Kohlenbach, On the computational content of the Krasnoselski and Ishikawa fixed point theorems, Proceedings of the FourthWorkshop on Computability and Complexity in Analysis, J. Blanck, V. Brattka, P. Hertling (eds.), Springer LNCS 2064 (2001) 119-145; https://doi.org/10.1007/3-540-45335-0

[8] U Kohlenbach, Uniform asymptotic regularity for Mann iterates, J. Math. Anal. Appl. 279 (2003) 531-544; https://doi.org/10.1016/S0022-247X(03)00028-3

[9] U Kohlenbach, Some logical metatheorems with applications in functional analysis, Trans. Amer. Math. Soc. 357 (2005) 89-128; https://www.jstor.org/stable/3845213

[10] U Kohlenbach, Applied Proof Theory: Proof Interpretations and their Use in Mathematics, Springer Monographs in Mathematics (2008); https://doi.org/10.1007/978-3540-77533-1

[11] U Kohlenbach, Recent Progress in Proof Mining in Nonlinear Analysis, IfCoLoG Journal of Logics and their Applications, Vol. 4, Issue 10 (2017) 3357-3406

[12] U Kohlenbach, A Koutsoukou-Argyraki, Rates of convergence and metastability for abstract Cauchy problems generated by accretive operators, J. Math. Anal. Appl. 423 (2015) 1089-1112; https://doi.org/10.1016/j.jmaa.2014.10.035

[13] U Kohlenbach, A Koutsoukou-Argyraki, Effective asymptotic regularity for oneparameter nonexpansive semigroups , J. Math. Anal. Appl. 433 (2016) 1883-1903; https://doi.org/10.1016/j.jmaa.2015.08.059

[14] A Koutsoukou-Argyraki, Proof Mining for Nonlinear Operator Theory: Four Case Studies on Accretive Operators, the Cauchy Problem and Nonexpansive Semigroups, PhD thesis (2017), URN: urn:nbn:de:tuda-tuprints-61015; http://tuprints.ulb.tudarmstadt.de/id/eprint/6101

[15] M A Krasnosel'skii, Two remarks on the method of successive approximations, Uspekhi Mat. Nauk, Vol. 10 (1955) 123-127; http://mi.mathnet.ru/umn7954

[16] G Kreisel, On the interpretation of non-finitist proofs, part I, Journal of Symbolic Logic, Vol. 16, (1951) 241-267; https://doi.org/10.1017/S0022481200100581

[17] G Kreisel, On the interpretation of non-finitist proofs, part II, Journal of Symbolic Logic, Vol. 17(1), (1952) 43-58; https://doi.org/10.2307/2267457

[18] P K F Kuhfittig, Common fixed points of nonexpansive mappings by iteration, Pacific J. Math. 97 (1981) 137-139; https://doi.org/10.2140/pjm.1981.97.137 
[19] L Leuştean, A quadratic rate of asymptotic regularity for CAT(0) spaces, J. Math. Anal. Appl. 325 (2007) 386-399; https://doi.org/10.1016/j.jmaa.2006.01.081

[20] T Suzuki, The set of common fixed points of a one-parameter continuous semigroup of mappings is $F(T(1)) \cap F(T(\sqrt{2}))$, Proceedings of the American Mathematical Society 134, No 3 (2005) 673-681; https://doi.org/10.1090/S0002-9939-05-08361-9

[21] T Suzuki, Common fixed points of one-parameter nonexpansive semigroups, Bull. London Math. Soc. 38 (2006) 1009-1018; https://doi.org/10.1112/S0024609306018893

Computer Laboratory, $15 \mathrm{JJ}$ Thomson Avenue, University of Cambridge CB3 OFD, UK

ak2110@cam.ac.uk

https://www.cl.cam.ac.uk/ ak2110/

Received: 8 March 2017 Revised: 16 August 2018 